\title{
A case of IgG4-related disease seemingly presenting as a T-cell lymphoma
}

\author{
Limei $\mathrm{Xu}^{1}$, Juan $\mathrm{Li}^{1}$, Ziyin $\mathrm{Ye}^{1}$, Xiaoyan Wang ${ }^{1}$, Hehua Wang ${ }^{1}$, Haihe Wang ${ }^{2}$, Xiuzhen Tong*1 \\ ${ }^{1}$ Department of Hematology, the First Affiliated Hospital, Sun Yat-sen University, Guangzhou, China \\ ${ }^{2}$ Department of Biochemistry, Zhongshan School of Medicine, Sun Yat-sen University, Guangzhou, China
}

Received: May 5, 2016

DOI: $10.5430 /$ crcp.v3n3p60
Accepted: June 20, 2016

Online Published: June 27, 2016

\begin{abstract}
IgG4-related disease (IgG4-RD) is an immune-mediated disorder hallmarked with lymphoplasmacytic tissues infiltrated with abundant IgG4-positive plasma cells, which has various clinical features and easily to be misdiagnosed. Here, we described a case with symptoms of body weight loss and multiple lymph node enlargements in a patient's groin, axilla, mediastinum, and retroperitoneum at the first admission. Both F-fluorodeoxyglucose (FDG)-position emission tomography (PET) and lymph node biopsies supported the possible diagnosis of this case as a T-cell lymphoma, but no TCR gene rearrangement observed. Further examinations showed that serum IgG4 level of the patient was elevated and large amount of IgG4+ plasmocyte infiltration was detected in the involved lymph nodes, indicating this case as a possible IgG4-RD. However, the possibility of this disease as a T-cell lymphoma still could not be excluded. Tentatively, the patient was first treated as IgG4-RD with oral prednisone combined with methotrexate to avoid the unnecessary side effect of chemotherapy and economy burden. After three weeks treatment, the serum IgG4 level of the patient is evidently decreased and the enlarged lymph nodes disappeared as well. Eventually, we characterized this case as an atypical IgG4-related lymphadenopathy with 20 months' follow-up.
\end{abstract}

Key Words: IgG4-related disease, Lymphadenopathy, IgG4, T-cell lymphoma

\section{INTRODUCTION}

IgG4-related disease (IgG4-RD) is an immune-mediated disorder hallmarked with the lymphoplasmacytic tissue infiltration with abundant IgG4-positive plasma cells and CD4+ $\mathrm{T}$ lymphocytes, generally accompanied by fibrosis, obliterative phlebitis, and elevated serum IgG4 level. ${ }^{[1]}$ IgG4$\mathrm{RD}$ can affect almost any organ, including lacrimal gland, pancreas, retroperitoneal clearance and so on. Patients with lymphadenopathy may manifest increased serum IgG4, serum $\operatorname{IgG}$ and $\operatorname{IgE}$, polyclonal hypergammaglobulinemia, and elevations in the erythrocyte sedimentation rate (ESR). The differential diagnosis in patients with generalized lymphadenopathy includes sarcoidosis, multicentric Castleman disease, infection (eg, tuberculosis), and lymphoma or other malignancy. IgG4-related lymphadenopathy is distinguished from these conditions by the modest lymph node enlargement, histologic distinctions on biopsy, lack of constitutional features, and the usually striking clinical response to glucocorticoids. ${ }^{[1]}$ When lack of other significant clinical manifestations, an IgG4-RD only presented with enlarged lymph nodes is often easily to be misdiagnosed as lymphoma though imaging examination or even pathological biopsy which is the most authoritative inspection for tumor. Here, we reported an IgG4-RD case that is presented as a pure lymphadenopathy at first admission with the possible symptom of T-cell lymphoma.

*Correspondence: Xiuzhen Tong; Email: tongxz05@163.com; Address: 58 Second Zhonshan Road, CPZN 510080, Guangzhou, China. 


\section{Case presentation}

A 56-year-old man initially presented to a local clinic with lacking in strength for two months, showing some painless masses in his bilateral inguinal, body weight loss and the low-grade fever. The routine blood test results were normal, but chest Computed Tomography (CT) scanning revealed the lymph nodes enlargements in patient's hilar, mediastinal and axillary areas, indicating the possibility of a lymphoma. Professional biopsy analysis of the right inguinal masses showed the symptom of lymphadenosis. To further clarify if it is a lymphoma, the F-fluorodeoxyglucose (FDG)-positron emission tomography (PET) was approached and manifested multiple enlarged lymph nodes in bilateral neck zone II, the left side of the neck region III, V, bilateral axillary, mediastinum, hilus pulmonis, abdominal compartment, bilateral lliac vessels side, and bilateral inguen, of which some lymph nodes inclined to mutual fusion with increased FDG uptake (SUVmax up to 11.4, while $<2$ in normal condition) (see Figure 1A). Given that a typical lymphoma has the all mentioned features, the patient was initially considered as lymphoma.
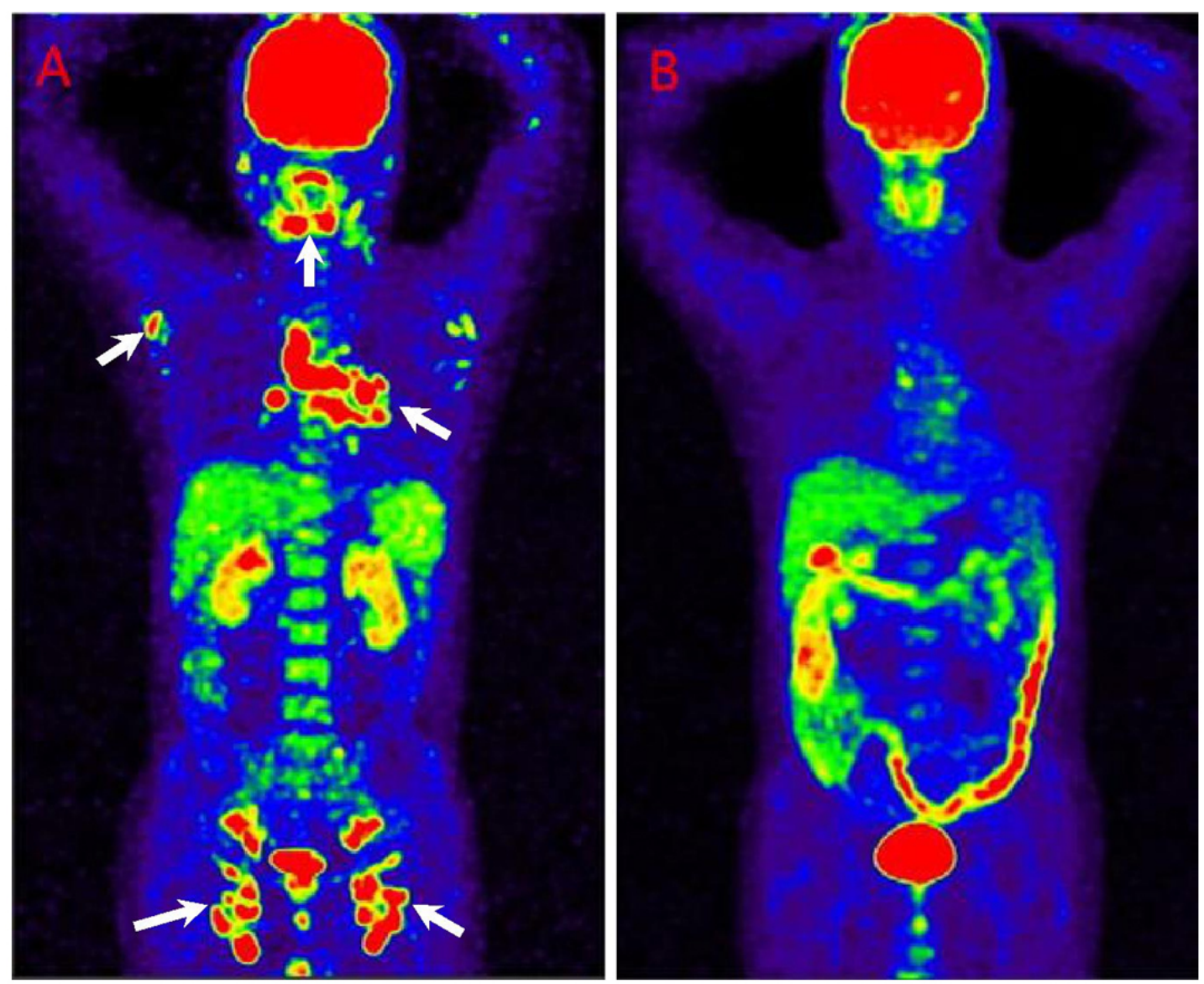

Figure 1. PET-CT images of the lymph nodes at the first admission and post 1 year's prednisone treatment A. Increased FDG uptake (SUVmax up to 11.4) was detected in multiple enlarged lymph nodes (Arrows pointed) at the first admission, including in bilateral necks, axillary, mediastinum, hilus pulmonis, abdominal compartment, bilateral lliac vessels side and bilateral inguinal folds. B. The sizes of lymph nodes were significantly reduced to normal, and the FDG uptake was also back to normal after one year's prednisone treatment, compared to that in Figure A.

The patient was transferred to our department with the worse symptom of weakness and progressive breathlessness, chest tightness and about $7 \mathrm{~kg}$ body weight loss at the time. But he did not presented any fever, mouth ulcers, skin rash, hair loss, joint pain and other discomfort. Physical examination showed that there were several painless enlarged lymph nodes in his groin, axilla, neck and the back of ears. The most enlarged lymph nodes were $2.3 \mathrm{~cm} \times 1.8 \mathrm{~cm}$ (right groin) in size. Other physical examination results were normal. Histopathological analyses of inguinal lymph node was carried out and revealed the disappearance of lymph-sinus, the thinner mantle zones with different thickness as destructive tendency (see Figure 2A) with diffusive infiltration by large amount of lymphoid cells, plasma cells and vessels in interfollicular areas (see Figure 2B). Immunohistochemical staining of the infiltrated lymph nodes showed that some 
lymphocytes were positive for T cell markers CD2, CD3, CD5 and CD7. Meanwhile, we especially observed abundant CD4+ and less CD8+ cells in interfollicular area, but no bcl-2, CD15 and CD56 positive cells; the Ki-67 labeled cells were about $40 \%$ in population; some plasma cells were positive for CD 138 and partially positive for $\kappa$ and $\lambda$, and
EBER detection showed a negative result. Histopathological examination of biopsy of axillary nodes was also conducted and showed similar findings. Based on all these observations, we tentatively characterized this case as an atypical lymphoid hyperplasia, especially like the peipheral T-cell lymphoma, not otherwise specified (PTCL-NOS). ${ }^{[2]}$
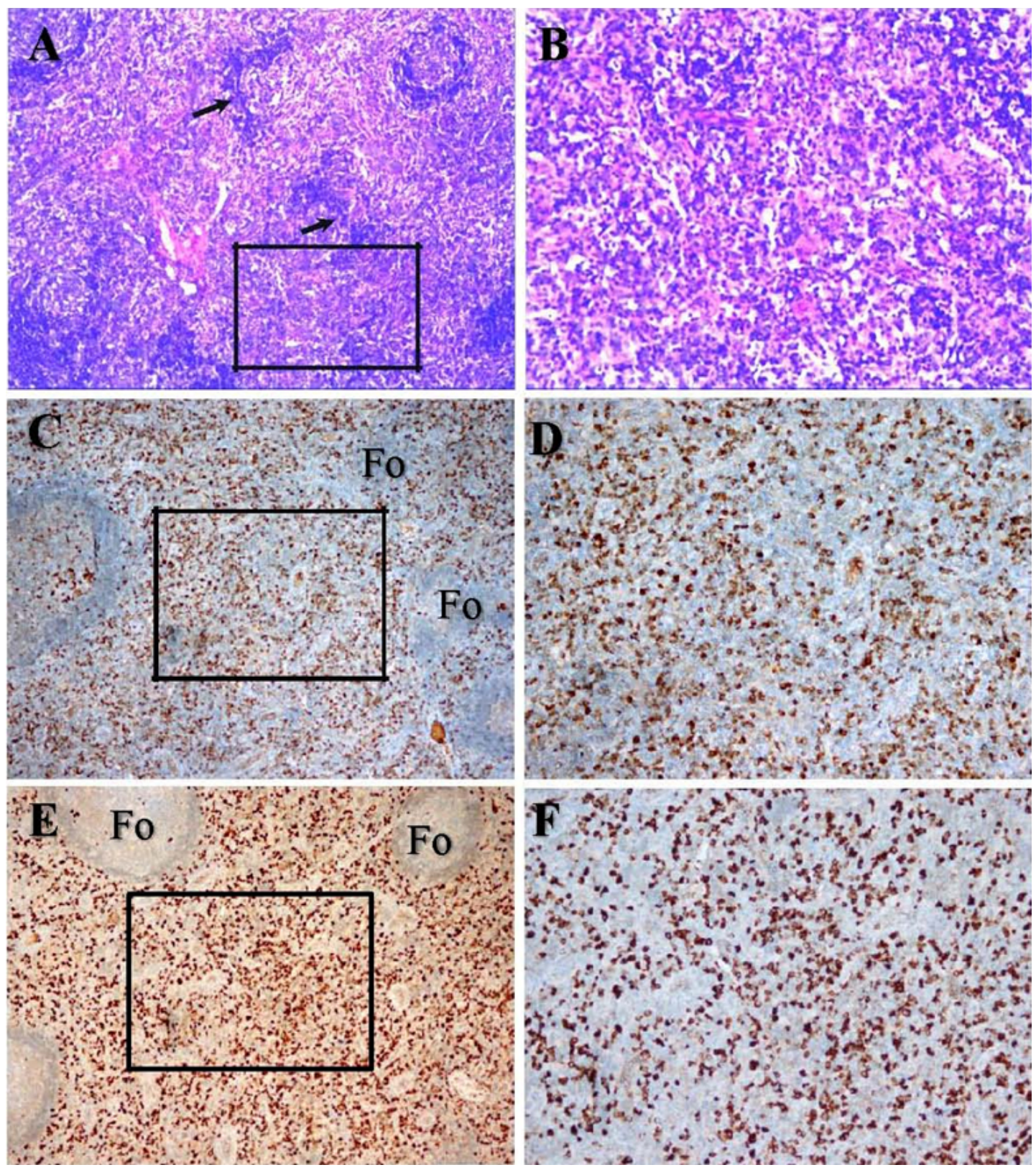

Figure 2. HE and IHC staining of the excised lymph nodes via biopsy

A. HE staining of the affected lymph nodes indicates the destructive tendency of lymph nodes, presenting as the disappearance of lymph-sinus, the thinner mantle zones with different thickness (indicated by arrows). Pictures were taken under $40 \times$ magnifications. B. Higher magnification observation of the region in Figure A shows diffusive infiltration by large amount of lymphoid cells, plasma cells and vessels in interfollicular area (indicated by arrows). Pictures were taken under $400 \times$ magnifications. $C$ and D, IHC staining of the affected lymph nodes with IgG antibody and shows numerous IgG+ cells in the interfollicular zones (Fo) under $40(C)$ and $400 \times(D)$ magnifications. E and F. IHC staining of the affected lymph nodes with IgG antibody and shows shows the IgG4+/IgG+ plasma cell ratio is $>40 \%$ in the interfollicular zones (Fo) under 40 (E) and $400 \times(F)$ magnifications. 
However, our gene rearrangement analyses unexpectedly showed that Immunoglobulin and TCR genes were normal. Blood tests also demonstrated the normal states of blood cells, alanine aminotransferase (ALT), aspartate aminotransferase (AST), and lactate dehydrogenase (LDH), but both anti-nuclear antibodies (ANA) and myeloperoxidase (MPO) were positive, and the ESR, serum amyloid A (SAA) and rheumatoid factor (RF) levels were increased. Serum IgG and $\mathrm{IgG} 4$ levels increased up to $40.30 \mathrm{~g} / \mathrm{L}$ (10.13-15.13 in normal) and $32.60 \mathrm{~g} / \mathrm{L}(\leq 2.00$ in normal) (see Table 1). All these information prompted us to further analyze the $\mathrm{IgG}$ profile by immunohistochemistry and the results showed there were abundant IgG positive plasma cells in the excisional lymph node specimen of the patient (see Figure 2C-D). IgG4 staining showed that the ratio of $\mathrm{IgG} 4+/ \mathrm{IgG}+$ positive plasma cells were at least more than 50\% of IgG-positive cells (see Figure 2E-F). Based on all above information, we assumed that this case could also be an IgG4-RD, although the possibility of this disease as a T-cell lymphoma can not be excluded.

Table 1. Overall response to prednisone treatment

\begin{tabular}{|c|c|c|c|c|c|}
\hline \multirow{2}{*}{ Time } & \multicolumn{2}{|c|}{ Treatment } & \multirow{2}{*}{$\begin{array}{c}\text { IgG4(mg/dl) } \\
\mathrm{nl} \leq 2\end{array}$} & \multirow{2}{*}{ LN swelling } & \multirow{2}{*}{ Chest CT } \\
\hline & Pred & MTX & & & \\
\hline 2014.07.07 & $35 \mathrm{mg}$ qd & 15mg H qw & 3260 & $\begin{array}{c}\text { post aurem, neck, } \\
\text { inguina }\end{array}$ & $\begin{array}{l}\text { multiple enlargement } \\
\text { lymph nodes in hilarregion, } \\
\text { mediastinal, axillary }\end{array}$ \\
\hline 2014.07.31 & $35 \mathrm{mg}$ qd & 15mg H qw & 1130 & NA & Smaller than before \\
\hline 2014.08.14 & 30mg qd & 15mg H qw & 934 & NA & Smaller than before \\
\hline 2014.08.28 & $25 \mathrm{mg}$ qd & 15mg H qw & 722 & NA & I \\
\hline 2014.10.17 & $20 \mathrm{mg}$ qd & 15mg H qw & 339 & NA & Smaller than before \\
\hline 2015.06.02 & $5 \mathrm{mg}$ qd & $15 \mathrm{mg} \mathrm{H} \mathrm{qw}$ & 449 & NA & Smaller than before \\
\hline
\end{tabular}

Note. Pred, prednisone; MTX, methotrexate; LN, lymph node; NA, not available; qw, once a week; qd, once a day; nl, normal. As shown in Table 1, the patient was followed up for almost one year with reduced hormone dosages and showed the satisfied health recovery.
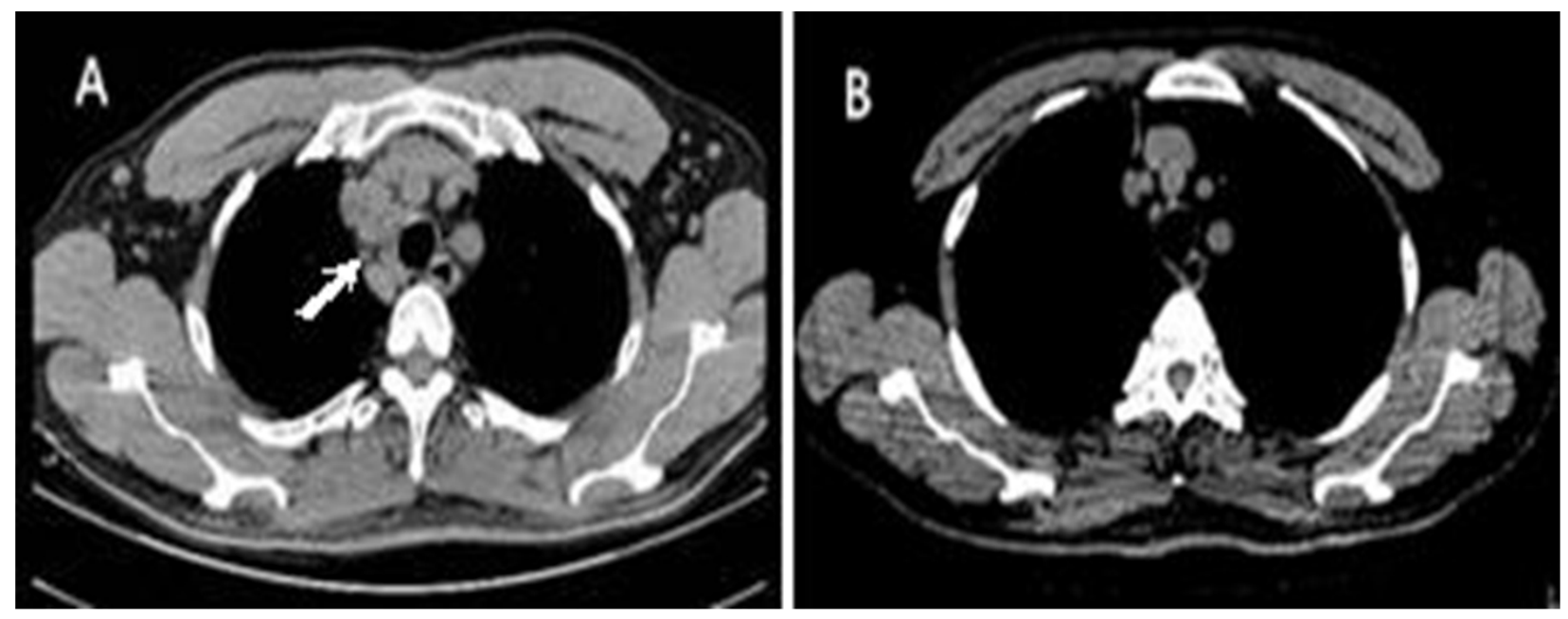

Figure 3. Chest CT scanning of lymph nodes in mediastina before and after 3 weeks' prednisone treatment A. CT scanning results demonstrating the enlarged lymph nodes in the mediastina (arrow pointed). B. CT scanning of the enlarged lymph nodes shows the significantly reduced mediastina after 3 weeks oral prednisone treatment.

Considering the harmful and expensive chemotherapy effects, we firstly suggested the tentative treatment of oral prednisone combined with methotrexate to monitor the immediate treatment response. After three weeks of treatment, the enlarged inguinal lymph nodes were completely disappeared (see Table 1). Further CT scanning showed the mediastinal lymph nodes evidently became smaller in size (see Figure 3). As shown in Table 1, we observed the gradual reduction of serum IgG4 level and the decreased lymph nodes in size. Thereafter, the patient was treated with gradually reduced hormone dosage under supervision (see Table 1). One year later, PET reexamination demonstrated that volumes of mediastinal, neck, bilateral axillary, abdominal cavity, bilateral iliac vessels paraneoplastic and bilateral inguinal lymph nodes were 
evidently reduced, and the FDG uptake was normal (see Figure 1B). During the follow up for 20 months, the patient was well without any discomfort. In view of his response to treatment, we eventually characterized this case as an atypical IgG4-related lymphadenopathy.

\section{Discussion}

IgG4-RD is a fibro-inflammatory condition within one or more organs and hallmarked with elevated level of circulating IgG4 in serum. ${ }^{[1]}$ As multiple organs, especially pancreas, bile ducts, salivary glands, lachrymal glands, mediastinal lymph nodes and retroperitoneum, could be affected, clinical presentation of IgG4-RD varies widely. ${ }^{[3]}$ Meanwhile, IgG4-RD also shows lymphadenectasis that sometimes simulate the first manifestation of other diseases and it is very easily to be misdiagnosed at first sight. Umehara et $\mathrm{al}^{[4]}$ described the diagnosis criteria for IgG4-RD as the followings: 1) clinical examination showing characteristic diffuse/localized swelling or masses in single or multiple organs, 2) hematological examination showing elevated serum IgG4 concentrations (135 mg/dl) and 3) histopathological examination showing the marked lymphocyte and plasmacyte infiltration and fibrosis, and IgG4+ plasma cells infiltration as IgG4+/IgG+ cells $>40 \%$ in ratio with $>10 \mathrm{IgG} 4+$ plasma cells/HPF. If a case fulfills to the criteria 1, 2 and 3, the case can be defined as IG4-RD, if 1 and 3, as probable, and 1 and 2 as possible, respectively. According to the above criteria, the case may be diagnosed as IgG4-RD, but the very common fibrosis in our case was not observed. Again, IgG4-RD often shows normal structure of lymph nodes, with no atypical lymphoid cells observed. Therefore, we cannot certain this case as IgG4-RD at this stage.

Sato Y et al, 2012 reported 40 cases of IgG4-related lymphadenopathy that were classified into the progressively transformed germinal center (PTGC) type, in which most cases are only with lymph node enlargement, and/or the elevated serum IgE levels as well as the marked eosinophil infiltration in the affected tissue and concomitant allergy; during follow-ups, $50 \%$ of the patients progressed to develop extranodal lesions in submandibular glands, lacrimal glands, mediastinals, kidneys or pancreases. ${ }^{[5]}$ In our case, the pathological presentation is totally distinct from the above mentioned subtypes, but presenting as the interfollicular expansion and immunoblastosis as well as the destructive tendencies of the lymph nodes interfollicular zone, which prompted us to link it to a T-cell lymphoma. Moreover, as known, all reported IgG4-RDs do not show the symptoms of fever, night sweat and body weight loss, which are often observed in lymphoma. ${ }^{[6]}$ In our case, we observed the obvious body weight loss and thus suspected that this case could be a lymphoma. In addition, some evidence indicates the correlations of IgG4 level elevation to lymphoma. For instances, several studies have reported that IgG4-RD can subsequently develop to non-hodgkin lymphoma(NHL) ${ }^{[7-10]}$ or IgG4-RD can conversely from lymphoma ${ }^{[11,12]}$ or IgG4-RD and lymphoma can simutaneously occur. ${ }^{[13]}$ Since IgG4-RD progressed to T-cell lymphoma is rare, ${ }^{[14]}$ our case indicates the possibility of coexistence of T-cell lymphoma and IgG4-RD.

Considering the T-cell receptor gene rearrangement is negative, we tentatively treated the patient with oral prednisone and got a favorable clinical response in a very short time, ruling out the simultaneous presences of IgG4-RD and Tcell lymphoma. The good response to glucocorticoid further proves the IgG4-RD nature of this case through our longterm follow-ups. We still will keep monitoring the disease progression in case of lymphoma occurrence.

\section{Conclusion}

In summary, taken all the morphological and pathological characteristics of lymph nodes together, we can classify our case into the type of interfollicular expansion and immunoblastosis as described prevously. ${ }^{[15]}$ Meanwhile, when imaging or even pathological biopsy cannot exclude the possibility of lymphoma for patients with lymph node enlargement, the examination of serum IgG4 level should be performed. To prevent harmful misdiagnosis and unnecessary chemotherapy, pathologists and clinicians should collaborate closely to evaluate each possibility of diseases. Further longterm clinical follow-up is crucial for the diagnosis of this disease when pathological examination cannot give a definite diagnosis.

\section{ACKNOWLEDGEMENTS}

This work was partially supported by Science and Technology Planning Project of Guangdong Province (No. 2013B021800127) and Natural Science Foundation of Guangdong (No.2015A030313090) to X. Tong.

\section{CONFLICTS OF INTEREST DisClOSURE}

The authors declare no conflicts of interest. 


\section{REFERENCES}

[1] Cheuk W, Chan JK. IgG4-related sclerosing disease: a critical appraisal of an evolving clinicopathologic entity. Adv Anat Pathol. 2010; 17: 303-32. PMid: 20733352. http://dx.doi.org/10.10 97/PAP. Ob013e3181ee63ce

[2] de Leval L, Gaulard P. Pathology and biology of peripheral T-cell lymphomas. Histopathology. 2011; 58: 49-68. PMid: 21261683. http://dx.doi.org/10.1111/j.1365-2559.2010.03704.x

[3] Palazzo E, Palazzo C, Palazzo M. IgG4-related disease. Joint Bone Spine. 2014; 81: 27-31. PMid: 23849464. http://dx.doi.org/1 $0.1016 / j \cdot j$ bspin.2013.06.001

[4] Umehara H, Okazaki K, Masaki Y, et al. Comprehensive diagnostic criteria for IgG4-related disease (IgG4-RD), 2011. Mod Rheumatol. 2012; 22: 21-30. PMid: 22218969. http://dx.doi.org/10.31 09/s10165-011-0571-z

[5] Sato Y, Inoue D, Asano N, et al. Association between IgG4-related disease and progressively transformed germinal centers of lymph nodes. Mod Pathol. 2012; 25: 956-67. PMid: 22481280. http: //dx.doi.org/10.1038/modpathol.2012.54

[6] Ansell SM. Non-Hodgkin Lymphoma: Diagnosis and Treatment. Mayo Clin Proc. 2015; 90: 1152-63. PMid: 26250731. http: //dx.doi.org/10.1016/j.mayocp. 2015.04.025

[7] Oyama T, Takizawa J, Nakamura N, et al. Multifocal mucosaassociated lymphoid tissue lymphoma associated with IgG4-related disease: a case report. Jpn J Ophthalmol. 2011; 55: 304-6. PMid: 21584726. http://dx.doi.org/10.1007/s10384-011 -0003-9

[8] Takahashi N, Ghazale AH, Smyrk TC, et al. Possible association between IgG4-associated systemic disease with or without autoimmune pancreatitis and non-Hodgkin lymphoma. Pancreas. 2009; 38: 523-6.
PMid: 19258916. http://dx.doi.org/10.1097/MPA.0b013e3 1819d73ca

[9] Marunaka H, Orita Y, Tachibana T, et al. Diffuse large B-cell lymphoma of the lacrimal sac arising from a patient with IgG4related disease. Mod Rheumatol. 2016; 1-5. PMid: 6708772. http: //dx.doi.org/10.3109/14397595.2015.1131353

[10] Sato Y, Takata K, Ichimura K, et al. IgG4-producing marginal zone B-cell lymphoma. Int J Hematol. 2008; 88: 428-33. PMid: 18839275. http://dx.doi.org/10.1007/s12185-008-0170-8

[11] Matsuo T, Ichimura K, Yoshino T. Local recurrence as immunoglobulin G4 (IgG4)-related disease 10 years after radiotherapy to ocular adnexal extranodal marginal zone B-cell lymphoma of mucosaassociated lymphoid tissue. J Clin Exp Hematop. 2011; 51: 125-33. PMid: 22104312. http://dx.doi.org/10.3960/jslrt.51.12 5

[12] Mitsui T, Yokohama A, Koiso H, et al. Development of IgG4-related disease 10 years after chemotherapy for diffuse large B cell lymphoma and longstanding bronchial asthma. Int J Hematol. 2013; 98: 122-8. PMid: 23666666. http://dx.doi.org/10.1007/s1218 5-013-1359-z

[13] Kim T, Grobmyer SR, Dixon LR, et al. Autoimmune pancreatitis and concurrent small lymphocytic lymphoma: not just a coincidence? J Gastrointest Surg. 2008; 12: 1566-70. PMid: 18506547. http://dx.doi.org/10.1007/s11605-008-0543-6

[14] Kanda G, Ryu T, Shirai T, et al. Peripheral T-cell lymphoma that developed during the follow-up of IgG4-related disease. Intern Med. 2011; 50: 155-60. PMid: 21245642. http://dx.doi.org/10.21 69/internalmedicine.50.4413

[15] Sato Y, Yoshino T. IgG4-Related Lymphadenopathy. Int J Rheumatol. 2012; 2012: 572539. http://dx.doi.org/10.1155/2012/5725 39 